\title{
On the Equivalence of the Massless DKP Equation and the Maxwell Equations in the Shuwer $¥ \oint$
}

\author{
Mustafa Saltı ${ }^{1} \|$ and Ali Havare ${ }^{2}$ \\ Department of Physics, Faculty of Art and Science, Mersin University \\ 33343-Ciftlikkoy, Mersin-Turkey \\ E-mail(s): ${ }^{1}$ musts6@yahoo.com, ${ }^{2}$ alihavare@mersin.edu.tr
}

\begin{abstract}
In this paper, a general relativistic wave equation is written to deal with electromagnetic waves in the background of the Shuwer. We obtain the exact form of this equation in a second order form. On the other hand, by using spinor form of the Maxwell equations the propagation problem is reduced to the solution of the second order differential equation of complex combination of the electric and magnetic fields. For these two different approach, we obtain the spinors in terms of field strength tensor. We show that the Maxwell equations are the equivalence with the mDKP equation in the Shuwer.
\end{abstract}

Keywords: Equivalence; Duffin-Kemmer-Petiau; Maxwell; General Relativity.

Pacs Numbers: 04.20.-q; 04.20.Cv

\section{Introduction}

Quantum field theory in curved space-time is one of the interesting sectors of the modern theoretical physics since it plays an important role to investigate the effects of the gravity in micro-particle world. To make out our real physical universe it is necessary to know the solutions of the both Einstein and quantum field equations to discuss the dynamics of the universe and its particles. The passage from the field equations of relativistic quantum mechanics in flat space-time to the general relativistic quantum field equations can be done by using the principle of covariance and the tetrad formalism according to the Tetrode-Weyl-Fock-Ivanenko procedure 1, 2, 3, 4, 5, 6, 7, 8, 9, expanded to include spin transformation quantities. Since the gravitational effects are weak it seems that general relativistic wave equations are not important in the atomic scale, but for many astrophysical situations one has to take into account gravitational effects due to their dominant role. One of the most fascinating aspects of the gravitational effects is their evident role in particle creation. To construct a steady theory of quantum field theory in

$\ddagger$ Mod. Phys. Lett. A, Vol.20 No.6, (2005) 451-465

$\S$ We refer to spatially homogenous universe models with expansion and rotation collectively as the Shuwer.

|| Corresponding Author. 
curved space-time it is necessary to analyze the single particle states since these states are examined to get the dynamics of the particles in a given background. The curved space-time quantum field theory provides a strong motivation for a unified theory of gravitation and quantum mechanics.

Historically, electromagnetic fields are described by the Maxwell equations. Following a minimal coupling procedure, scalar products are performed with the Riemannian metric $g_{\mu \nu}$, and the partial derivatives are replaced covariant ones, the Maxwell equations can be written in general relativity [10. In the literature, however, there exist many attempts to pass down from the classical wave theory of light to quantum mechanics 11. If complex combinations of the electric and magnetic fields are taken as the elements of a three-component spinor, the Maxwell curl equations can be synthesized into a form similar to that of the Weyl equation for the neutrino. In these three-component formulation the divergence equations are imposed as constraint equations. Furthermore, these are valid only in free space in the absence of any source. These two deficiencies have been put right in the work of Moses 12, who found a four-component spinor formulation which casts the Maxwell equations in the form of a massless Dirac equation. He united the source in the form of a four-component spinor and combined the four Maxwell equations in the presence of a source.

Much earlier than the above attempts, Duffin, Kemmer and Petiau had formulated the wave equation(the DKP equation) for massive spin-1 particles [13, 14. They showed that the first order form of the Klein-Gordon and Proca field equations can be represented in the Dirac-like matrix form

$$
\left(i \beta^{(k)} \partial_{(k)}-m\right) \Psi=0
$$

where $\beta$ matrices satisfy the following relation

$$
\beta^{(a)} \beta^{(b)} \beta^{(c)}+\beta^{(c)} \beta^{(b)} \beta^{(a)}=\beta^{(a)} \delta^{(b)(c)}+\beta^{(c)} \delta^{(b)(a)}
$$

Equation (11) is a first order equation for spin-0 and spin-1 bosons, in contrast to the other relativistic wave equations for bosons. Lately, the applications of the Duffin-KemmerPetiau (DKP) theory to Quantum Chromo-dynamics (QCD) have been considered by Gribov [15. Additionally, it has been used to find covariant Hamiltonian dynamics by Kanatchikov[16]. Within the framework of general relativity the DKP equation has been conformed to curved space-time by Red'kov[17] and Lunardi et al. 18. With the generalization of DKP equation to the curved space-time it has become important to investigate the behavior of bosons in curved backgrounds. The covariant form of DKP equation is given by

$$
\left(i \beta^{\mu} \nabla_{\mu}-m\right) \Psi=0
$$

where

$$
\beta^{\mu}(x)=\gamma^{\mu}(x) \otimes \mathrm{I}+\mathrm{I} \otimes \gamma^{\mu}(x)
$$

are the Kemmer matrices in curved space-time and they are related to flat Minkowski space-time as $\beta^{\mu}(x)=b_{(i)}^{\mu} \widetilde{\beta}^{(i)}$ with a tetrad frame that satisfies $g_{\mu \nu}=b_{\mu}^{(i)} b_{\nu}^{(j)} \eta_{(i)(j)}$. The 
covariant derivative in equation (3) is $\nabla_{\mu}=\partial_{\mu}-\Omega_{\mu}$ with spinorial connections which can be written as

$$
\Omega_{\mu}=\Gamma_{\mu} \otimes I+I \otimes \Gamma_{\mu}
$$

where

$$
4 \Gamma_{\lambda}=g_{\mu \alpha}\left[\left(\partial_{\lambda} a_{\nu}^{(k)}\right) b_{(k)}^{\alpha}-\Gamma_{\nu \lambda}^{\alpha}\right] S^{\mu \nu}
$$

with Christoffel symbols and spin tensor which can be written as

$$
\Gamma_{\mu \nu}^{\alpha}=\frac{1}{2} g^{\alpha \beta}\left(\partial_{\mu} g_{\beta \nu}+\partial_{\nu} g_{\beta \mu}-\partial_{\beta} g_{\mu \nu}\right), \quad S^{\mu \nu}=\left[\gamma^{\mu}, \gamma^{\nu}\right]
$$

where $\Gamma_{\mu \nu}=\Gamma_{\nu \mu}$ and $\gamma^{\mu}$ are the Dirac matrices in curved space-time and they're related to flat Minkowski space-time as

$$
\gamma^{\mu}(x)=b_{(i)}^{\mu} \widetilde{\gamma}^{(i)}
$$

The Dirac-like equation (3) can be solved by using standard techniques used for the Dirac equation.

The counterpart of the Maxwell equations in general relativistic quantum mechanics can be obtained as the zero-mass limit of the DKP equation with appropriate identification of the components of the DKP spinor with electromagnetic field strengths. In 1997 Ünal showed that the wave equation of massless spin-1 particle in flat space-time is equivalent to free space Maxwell equations [19]. Then Ünal and Sucu solved the general relativistic massless DKP equations (hereafter referred to as the mDKP equation) in Robertson-Walker space-time written in spherical coordinates[20]. By using the same technique the mDKP equation had been solved for the stationary Gödel and the Gödeltype space-time and also the non-stationary Gödel-type cosmological universes [21, 22]. In this technique the Kemmer matrices are written as a direct product of Pauli spin matrices with unit matrix resulting $(4 \times 4)$ matrices. This representation leads to a spinor which is related to complex combination of the electric and magnetic fields. Among of the advantages to use the mDKP equation is that its simple $(4 \times 4)$ matrix form simplify the solution procedure for comparing with the Maxwell equations. As well the Quantum mechanical solution is important in a discussion of the wave-particle duality of electromagnetic fields, since the particle nature of the electromagnetic field can be analyzed only by a quantum mechanical equation. Furthermore, the mDKP equation removes the unavoidable usage of $(3+1)$ space-time splitting formalism for the Maxwell equations mentioned by Saibatalov[23]. The mDKP equation is given as follow

$$
\beta^{\mu} \nabla_{\mu} \Psi=0
$$

where $\beta^{\mu}$ are now:

$$
\beta^{\mu}(x)=\sigma^{\mu}(x) \otimes \mathrm{I}+\mathrm{I} \otimes \sigma^{\mu}(x)
$$

with

$$
\sigma^{\mu}(x)=(\mathrm{I}, \vec{\sigma}(x))
$$


The covariant derivative $\nabla_{\mu}$ with spinorial connections $\Xi_{\mu}$ are given with the limit $\gamma^{\mu} \rightarrow \sigma^{\mu}$ as

$$
\nabla_{\mu}=\partial_{\mu}-\Xi_{\mu}=\partial_{\mu}-\lim _{\gamma \rightarrow \sigma} \Gamma_{\mu} \otimes I+I \otimes \Gamma_{\mu}
$$

In this paper, we investigate the relationship between the classical and quantum theory of light by examining mDKP equation and the Maxwell equations in the Shuwer. In that manner we show quantum mechanical wave function in terms of the Maxwell field strength tensor components. Since $4 \times 4$ Kemmer matrices have been used this correspondence can be shown only if a complex combination of the field strength tensors are used. This paper is organized as follow: in the next section we define a metric which is describe the Shuwer and calculate some kinematics in this cosmological models. In section III, we give the mDKP equation explicitly and we obtain its second order form for a given geometry. In section IV, we find the components of the Maxwell field strengths and use them to get second order differential equation by using spinor formalism in the Shuwer. In section $\mathrm{V}$, we obtain oscillating frequency of the photon in some special limits , and at the last section, we give some results and discussions.

\section{The Spatially Homogenous Universes with Expansion and Rotation}

The Shuwer's line element which we chosen is given by

$$
d s^{2}=-g_{00}\left(x^{0}, x^{1}\right)\left(d x^{0}\right)^{2}+g_{i i}\left(x^{0}, x^{1}\right)\left(d x^{i}\right)^{2}-2 g_{02}\left(x^{0}, x^{1}\right) d x^{0} d x^{2}
$$

where $i=1,2,3$.

The line element (13) can be reduces to known space-time models under some conditions. We give some space-times which have the special cases given in table-1 which are the form in $(3+1)$-dimensional non-diagonal of the line element (13).

Here $\alpha, \lambda, a, c, \omega_{0}, r_{0}, B$ are constants. In table- 2 we give various well-known spacetimes which are included by the line-element (13). Defining $A, \sigma, a, b, c$, are constants and

$$
\Lambda=\left(1+\frac{B^{2}}{4} \rho^{2}\right)
$$

then following line-elements can be written as given by table-2.

Considering the special space-times given in table- 1 and table- 2 then the line element (13) could be written as

$$
d s^{2}=-R_{00}^{2}\left(x^{1}\right)\left(d x^{0}\right)^{2}+T^{2}\left(x^{0}\right) R_{i i}^{2}\left(x^{1}\right)\left(d x^{i}\right)^{2}-2 T\left(x^{0}\right) R_{02}\left(x^{1}\right) d x^{0} d x^{2}
$$

where $i=1,2,3$. From this point of view if we make the following coordinate transformation

$$
d \tilde{x}^{m}=R_{m m} d x^{m}
$$

here $(m=0,1,3)$ and defining $\tilde{x}^{m}=(\eta, \xi, u), G=\frac{R_{02}}{R_{00}}$ then the line element (13) takes the form:

$$
d s^{2}=-d \eta^{2}+T^{2}(\eta)\left[d \xi^{2}+R_{22}^{2}(\xi)\left(d x^{2}\right)^{2}+d u^{2}\right]-2 T(\eta) G(\xi) d \eta d x^{2}
$$


Table 1. (3+1)-dimensional non-diagonal line elements:

\begin{tabular}{ll}
\hline Space-times & Line-elements \\
\hline Gödel[21] & $-\left(d t+e^{\alpha r} d \theta\right)^{2}+d r^{2}+\frac{1}{2}\left(e^{\alpha r} d \theta\right)^{2}+d z^{2}$ \\
Expanding Gödel-type[26] & $c^{2} t^{2}\left(d x^{2}+\lambda e^{2 m x} d y^{2}+d z^{2}\right)-2 c t e^{m x} d t d y-d t^{2}$ \\
Reboucas[26] & $d r^{2}-\left(1+3 \cosh ^{2} 2 r\right) d \phi^{2}+d z^{2}+4 \cosh 2 r d t d \phi-d t^{2}$ \\
Som-Raychaudhuri[26] & $d r^{2}+r^{2}\left(1-r^{2}\right) d \phi^{2}+d z^{2}-2 r^{2} d t d \phi-d t^{2}$ \\
Hoenselaers-Vishveshwara [26] & $d r^{2}-\frac{1}{2}(\operatorname{coshr}-1)(\operatorname{coshr}-3) d \phi^{2}+d z^{2}-2(\operatorname{coshr}-1) d t d \phi-d t^{2}$ \\
Krechet-Gödel[27] & $R^{2}(t)\left[d x^{2}-\frac{1}{2} e^{2 \sqrt{2} \omega_{0} x} d y^{2}+d u^{2}\right]+2 R(t) e^{\sqrt{2} \omega_{0} x} d t d y-d t^{2}$ \\
Gödel-Friedman[28] & $a^{2}\left[-d \eta^{2}+d \varepsilon^{2}+d y^{2}+\sinh h^{2} \varepsilon\left(1-\sinh h^{2} \varepsilon\right) d \phi^{2}-2 \sqrt{2} \sinh h^{2} \varepsilon d \eta d \phi\right.$ \\
Soleng[29] & $d r^{2}-2 a d t d \phi+\left(B^{2}\left(r+r_{0}\right)^{2}-a^{2}\right) d \phi^{2}+d z^{2}-d t^{2}$ \\
Weyl-Levis-Papapetrou[ $[0]$ & $-e^{2 U(\rho)}(d t+a d \phi)^{2}+e^{-2 U(\rho)}\left[e^{2 k}\left(d \rho^{2}+d \zeta^{2}\right)+\rho^{2} d \phi^{2}\right]$ \\
\hline
\end{tabular}

Table 2. (3+1)-dimensional diagonal line elements:

\begin{tabular}{ll}
\hline Space-times & Line-elements \\
\hline Robertson-Walker [26] & $R^{2}(t)\left(d x^{2}+d y^{2}+d z^{2}\right)-d t^{2}$ \\
Minkowski[26] & $d x^{2}+d y^{2}+d z^{2}-d t^{2}$ \\
Weyl[30] & $-e^{2 U(r)} d t^{2}+e^{2[K(r)-U(r)]}\left(d r^{2}+d z^{2}\right)+r^{2} e^{-2 U(r)} d \phi^{2}$ \\
Kantowski-Sachs[31] & $d x^{2}+\sin ^{2} x d y^{2}+d z^{2}-d t^{2}$ \\
Bianchi type-V[31] & $d x^{2}+e^{2 x}\left(d y^{2}+d z^{2}\right)-d t^{2}$ \\
Bianchi type-VI(A) [31] & $d x^{2}+e^{2(A-1) x} d y^{2}+e^{(A+1) x} d z^{2}-d t^{2}$ \\
Bianchi type-VII(A)[31] & $d x^{2}+e^{2 A x}\left(d y^{2}+d z^{2}\right)-d t^{2}$ \\
Melvin[32] & $\rho^{2} \Lambda^{-2} d \phi^{2}+\Lambda^{2}\left(-d T^{2}+d Z^{2}+d \rho^{2}\right)$ \\
Rindler Flat[33] & $-x^{2} d t^{2}+d x^{2}+d y^{2}+d z^{2}$ \\
Levi-Civita[33] & $-r^{4 \sigma} d t^{2}+r^{4 \sigma(2 \sigma-1)}\left(d r^{2}+\frac{1}{a^{2}} d m^{2}\right)+\frac{1}{b^{2}} r^{2(1-2 \sigma)} d n^{2}$ \\
Cydl. Sym. Minkowski[34] & $d r^{2}+r^{2} d \phi^{2}+d z^{2}-d t^{2}$ \\
Flat Plate[34] & $-(a+b x)^{2} d t^{2}+d x^{2}+c\left(d y^{2}+d z^{2}\right)$ \\
A General Static[35] & $\left(r^{2}-a\right)\left(d z^{2}-d t^{2}\right)+d r^{2}+\left(b-r^{2}\right) d \phi^{2}$ \\
\hline
\end{tabular}


Table 3. List of the kinematics and their formulas:

\begin{tabular}{ll}
\hline Kinematics & Formulas \\
\hline four-acceleration vector: & $a_{i}=c_{i 0}^{0}$ \\
vorticity tensor: & $\omega_{i j}=\frac{1}{2} c_{i j}^{0}$ \\
expansion(deformation) tensor: & $\theta_{i j}=\frac{1}{2}\left(c_{i 0 j}+c_{j 0 i}\right)$ \\
expansion scalar: & $\theta=c_{01}^{1}+c_{02}^{2}+c_{03}^{3}$ \\
vorticity vector: & $\omega^{1}=\frac{1}{2} c_{23}^{0}, \quad \omega^{2}=\frac{1}{2} c_{31}^{0}, \quad \omega^{3}=\frac{1}{2} c_{12}^{0}$ \\
vorticity scalar: & $\omega^{2}=\frac{1}{4}\left[\left(c_{23}^{0}\right)^{2}+\left(c_{31}^{0}\right)^{2}+\left(c_{12}^{0}\right)^{2}\right]$ \\
shear tensor: & $\sigma_{i j}=\theta_{i j}-\frac{1}{3} \theta \delta_{i j}$ \\
\hline
\end{tabular}

This metric describes spatially homogenous universes with expansion and rotation, but zero shear. The matrices of the $g_{\mu \nu}$ and $g^{\mu \nu}$ are defined by

$$
\left(\begin{array}{cccc}
-1 & 0 & -T G & 0 \\
0 & T^{2} & 0 & 0 \\
-T G & 0 & T^{2} R_{22}^{2} & 0 \\
0 & 0 & 0 & T^{2}
\end{array}\right),\left(\begin{array}{cccc}
-\frac{R_{22}^{2}}{M^{2}} & 0 & -\frac{G}{T M^{2}} & 0 \\
0 & \frac{1}{T^{2}} & 0 & 0 \\
-\frac{G}{T M^{2}} & 0 & \frac{1}{M^{2} T^{2}} & 0 \\
0 & 0 & 0 & \frac{1}{T^{2}}
\end{array}\right)
$$

where $M^{2}=G^{2}+R_{22}^{2}$.

After the pioneering works of Gamow 24] and Gödel [25], the idea of global rotation of the universe has became considerably important physical aspect in the calculations of general relativity. For the Shuwer, one can introduces the tetrad basis as follow:

$$
\theta^{0}=d \eta+T G d x^{2}, \quad \theta^{1}=T d \xi, \quad \theta^{2}=T M d x^{2}, \quad \theta^{3}=T d u .
$$

where $M^{2}=G^{2}+R_{22}^{2}$. By use of the co-moving tetrad formalism, the kinematics of this model can be expressed solely in terms of the structure coefficients of the tetrad basis. We define the structure coefficients by

$$
d \theta^{\alpha}=\frac{1}{2} c_{\beta \gamma}^{\alpha} \theta^{\beta} \wedge \theta^{\gamma}
$$

By taking the exterior derivatives of the tetrad basis (19) and using the kinematics formulas [27]:

we find the following quantities for the line element

$$
a_{2}=\frac{\dot{T} G}{T M}, \quad \omega^{3}=\frac{G^{\prime}}{2 T M}, \quad \theta_{i j}=\frac{\dot{T}}{T} \delta_{i j}, \quad \sigma_{i j}=0 .
$$

where dot and prime indicates derivative with respect to $\eta$ and $\xi$ respectively. The model given in (17) is shear-free expansion and non-vanishing vorticity. We also note that this frame has a non-vanishing four-acceleration when $\dot{T} \neq 0$. 


\section{The Second Order Form of the mDKP Equation}

For the line element given in equation (17) the suitably selected tetrads are

$$
\begin{array}{llll}
a_{\mu}^{(0)}=\delta_{\mu}^{0}, & a_{\mu}^{(1)}=T \delta_{\mu}^{1}, & a_{\mu}^{(2)}=T\left(G \delta_{\mu}^{0}+M \delta_{\mu}^{2}\right), & a_{\mu}^{(3)}=T \delta_{\mu}^{3} \\
b_{(0)}^{\mu}=\delta_{0}^{\mu}, & b_{(1)}^{\mu}=\frac{1}{T} \delta_{1}^{\mu}, & b_{(2)}^{\mu}=\frac{1}{M}\left(\frac{1}{T} \delta_{2}^{\mu}-G \delta_{0}^{\mu}\right), & b_{(3)}^{\mu}=\frac{1}{T} \delta_{3}^{\mu}
\end{array}
$$

where $a_{(i)}^{\mu}=g^{\mu \nu} \eta_{(i)(j)} b_{\nu}^{(j)}$. The curved Dirac matrices which satisfy $\gamma^{\mu}, \gamma^{\nu}=2 g^{\mu \nu}$ are given by

$$
\gamma^{\eta}=\widetilde{\gamma}^{\eta}-\frac{G}{M} \widetilde{\gamma}^{2}, \quad \gamma^{\xi}=\frac{1}{T} \widetilde{\gamma}^{\xi}, \quad \gamma^{2}=\frac{1}{T M} \widetilde{\gamma}^{2}, \quad \gamma^{u}=\frac{1}{T} \widetilde{\gamma}^{u} .
$$

The Christoffel symbols are

$$
\begin{aligned}
& M^{2} \Gamma_{\mu \nu}^{0}=\dot{T} G^{2} T^{-1} \delta_{\mu \nu}^{00}+R_{22}^{2}\left[T\left(\delta_{\mu \nu}^{11}+R_{22}^{2} \delta_{\mu \nu}^{22}+\delta_{\mu \nu}^{33}\right)-G\left(\delta_{\mu \nu}^{02}+\delta_{\mu \nu}^{20}\right)\right] \\
& +\frac{G G^{\prime}}{2}\left(\delta_{\mu \nu}^{01}+\delta_{\mu \nu}^{10}\right)+\frac{T R_{22}}{2}\left(R_{22} G^{\prime}-2 G R_{22}^{\prime}\right)\left(\delta_{\mu \nu}^{12}+\delta_{\mu \nu}^{21}\right) \\
& T \Gamma_{\mu \nu}^{1}=\dot{T}\left(\delta_{\mu \nu}^{01}+\delta_{\mu \nu}^{10}\right)+\frac{G^{\prime}}{2}\left(\delta_{\mu \nu}^{02}+\delta_{\mu \nu}^{20}\right)-T R_{22} R_{22}^{\prime} \delta_{\mu \nu}^{22} \\
& M^{2} \Gamma_{\mu \nu}^{2}=\dot{T} G\left(\delta_{\mu \nu}^{11}-T^{-2} \delta_{\mu \nu}^{00}+R_{22}^{2} \delta_{\mu \nu}^{22}+\delta_{\mu \nu}^{33}\right)+R_{22}^{2} T^{-1}\left(\delta_{\mu \nu}^{02}+\delta_{\mu \nu}^{20}\right) \\
& -\frac{G^{\prime}}{2 T}\left(\delta_{\mu \nu}^{01}+\delta_{\mu \nu}^{10}\right)+\frac{1}{2}\left(G G^{\prime}+2 R_{22} R_{22}^{\prime}\right)\left(\delta_{\mu \nu}^{12}+\delta_{\mu \nu}^{21}\right)
\end{aligned}
$$

and

$$
T \Gamma_{\mu \nu}^{3}=\dot{T}\left(\delta_{\mu \nu}^{03}+\delta_{\mu \nu}^{30}\right)
$$

where $\delta_{\mu \nu}^{\alpha \beta}=\delta_{\mu}^{\alpha} \delta_{\nu}^{\beta}$ and the spinorial connections are

$$
\begin{aligned}
& \Gamma_{0}=\frac{G \dot{T}}{2 T M} \widetilde{\gamma}^{2 \eta}-\frac{G^{\prime}}{4 T M} \widetilde{\gamma}^{\xi 2}, \quad \Gamma_{3}=\frac{-\dot{T}}{2} \widetilde{\gamma}^{u \eta}-\frac{G \dot{T}}{2 M} \widetilde{\gamma}^{2 u} \\
& \Gamma_{1}=\frac{-\dot{T}}{2} \widetilde{\gamma}^{\xi \eta}+\frac{G \dot{T}}{2 M} \widetilde{\gamma}^{\xi 2}+\frac{G^{\prime}}{4 M} \widetilde{\gamma}^{2 \eta} \\
& \Gamma_{2}=\frac{-G^{\prime}}{4} \widetilde{\gamma}^{\xi \eta}-\frac{R_{22}^{2} \dot{T}}{2 M} \widetilde{\gamma}^{2 \eta}+\frac{G G^{\prime}+2 R_{22} R_{22}^{\prime}}{4 M} \widetilde{\gamma}^{\xi 2}
\end{aligned}
$$

here $\widetilde{\gamma}^{i j}=\widetilde{\gamma}^{i} \widetilde{\gamma}^{j}$. Using standard representation of the Dirac matrices we obtain the mDKP equation as

$$
\begin{aligned}
\left(\widetilde{\beta}^{\eta}-\right. & \left.\frac{G}{M} \widetilde{\beta}^{2}\right) \partial_{\eta} \Psi+\frac{1}{T} \widetilde{\beta}^{\xi} \partial_{\xi} \Psi+\frac{1}{T M} \widetilde{\beta}^{2} \partial_{2} \Psi+\frac{1}{T} \widetilde{\beta}^{u} \partial_{u} \Psi-\frac{G \dot{T}}{2 T M} \widetilde{\beta}^{\eta 2} \Psi \\
& +\frac{i G^{\prime}}{4 T M} \widetilde{\beta}^{\eta u} \Psi-\frac{i M^{\prime}}{2 T M} \widetilde{\beta}^{2 u} \Psi-\frac{i G \dot{T}}{2 T M}\left(\widetilde{\beta}^{\xi u}-\widetilde{\beta}^{u \xi}\right) \Psi \\
& +\frac{\dot{T}}{2 T}\left[\left(\widetilde{\beta}^{\xi}\right)^{2}+\left(\widetilde{\beta}^{2}\right)^{2}+\left(\widetilde{\beta}^{u}\right)^{2}\right] \Psi-\frac{G^{\prime}}{4 T M}\left(\widetilde{\beta}^{\xi 2}-\widetilde{\beta}^{2 \xi}\right) \Psi=0
\end{aligned}
$$


where $\widetilde{\beta}^{i j}=\widetilde{\beta}^{i} \widetilde{\beta}^{j}$. If the following spinor definition is used

$$
\Psi=\left(\begin{array}{c}
\Psi_{0} \\
\Psi_{1} \\
\Psi_{2} \\
\Psi_{3}
\end{array}\right)
$$

equation (30) gives four coupled first order differential equations in terms of the components of the spinor as

$$
\begin{aligned}
& \left(2 \partial_{\eta}+\frac{2}{T} \partial_{u}+\frac{4 \dot{T}}{T}\right) \Psi_{0}+\left(\frac{i G}{M} \partial_{\eta}+\frac{1}{T} \partial_{\xi}-\frac{i}{T M} \partial_{2}+\frac{2 i G \dot{T}}{T M}\right)\left(\Psi_{1}+\Psi_{2}\right)=0( \\
& \left(\frac{-i G}{M} \partial_{\eta}+\frac{1}{T} \partial_{\xi}+\frac{i}{T M} \partial_{2}-\frac{2 i G \dot{T}}{T M}+\frac{M^{\prime}}{T M}\right) \Psi_{0}+\left(2 \partial_{\eta}+\frac{2 \dot{T}}{T}\right) \Psi_{1} \\
& +\frac{2 \dot{T}}{T} \Psi_{2}+\left(\frac{i G}{M} \partial_{\eta}+\frac{1}{T} \partial_{\xi}-\frac{i}{T M} \partial_{2}+\frac{2 i G \dot{T}}{T M}+\frac{M^{\prime}}{T M}\right) \Psi_{3}=0 \\
& \left(\frac{-i G}{M} \partial_{\eta}+\frac{1}{T} \partial_{\xi}+\frac{i}{T M} \partial_{2}-\frac{2 i G \dot{T}}{T M}+\frac{M^{\prime}}{T M}\right) \Psi_{0}+\frac{2 \dot{T}}{T} \Psi_{1} \\
& +\left(2 \partial_{\eta}+\frac{2 \dot{T}}{T}\right) \Psi_{2}+\left(\frac{i G}{M} \partial_{\eta}+\frac{1}{T} \partial_{\xi}-\frac{i}{T M} \partial_{2}+\frac{2 i G \dot{T}}{T M}+\frac{M^{\prime}}{T M}\right) \Psi_{3}=0 \\
& \left(\frac{-i G}{M} \partial_{\eta}+\frac{1}{T} \partial_{\xi}+\frac{i}{T M} \partial_{2}-\frac{2 i G \dot{T}}{T M}\right)\left(\Psi_{1}+\Psi_{2}\right)+\left(2 \partial_{\eta}-\frac{2}{T} \partial_{u}+\frac{4 \dot{T}}{T}\right) \Psi_{3}=0(
\end{aligned}
$$

from equations (33) and (34) it's seen that $\Psi_{1}=\Psi_{2}$. If we use this result then these four coupled equations reduce to following three coupled equations:

$$
\begin{aligned}
& \left(\partial_{\eta}+\frac{1}{T} \partial_{u}+\frac{2 \dot{T}}{T}\right) \Psi_{0}+\left(\frac{i G}{M} \partial_{\eta}+\frac{1}{T} \partial_{\xi}-\frac{i}{T M} \partial_{2}+\frac{2 i G \dot{T}}{T M}\right) \Psi_{1}=0 \\
& \left(\frac{-i G}{M} \partial_{\eta}+\frac{1}{T} \partial_{\xi}+\frac{i}{T M} \partial_{2}-\frac{2 i G \dot{T}}{T M}+\frac{M^{\prime}}{T M}\right) \Psi_{0}+\left(2 \partial_{\eta}+\frac{4 \dot{T}}{T}\right) \Psi_{1} \\
& \quad+\left(\frac{i G}{M} \partial_{\eta}+\frac{1}{T} \partial_{\xi}-\frac{i}{T M} \partial_{2}+\frac{2 i G \dot{T}}{T M}+\frac{M^{\prime}}{T M}\right) \Psi_{3}=0 \\
& \left(\frac{-i G}{M} \partial_{\eta}+\frac{1}{T} \partial_{\xi}+\frac{i}{T M} \partial_{2}-\frac{2 i G \dot{T}}{T M}\right) \Psi_{1}+\left(\partial_{\eta}-\frac{1}{T} \partial_{u}+\frac{2 \dot{T}}{T}\right) \Psi_{3}=0 .
\end{aligned}
$$

If the following conformal time and spinor definition are used

$$
\tau=\int T^{-1}(\eta) d \eta, \quad \Psi=T^{-2} \phi
$$

then we get

$$
\begin{aligned}
& \left(\partial_{\tau}+\partial_{u}\right) \phi_{0}+\left(\frac{i G}{M} \partial_{\tau}+\partial_{\xi}-\frac{i}{M} \partial_{2}\right) \phi_{1}=0 \\
& \left(\frac{-i G}{M} \partial_{\tau}+\partial_{\xi}+\frac{i}{M} \partial_{2}+\frac{M^{\prime}}{M}\right) \phi_{0}+2 \partial_{\tau} \phi_{1}+\left(\frac{i G}{M} \partial_{\tau}+\partial_{\xi}-\frac{i}{M} \partial_{2}+\frac{M^{\prime}}{M}\right) \phi_{3}=0 \\
& \left(\frac{-i G}{M} \partial_{\tau}+\partial_{\xi}+\frac{i}{M} \partial_{2}\right) \phi_{1}+\left(\partial_{\tau}-\partial_{u}\right) \phi_{3}=0
\end{aligned}
$$


for these three coupled equations we can choose the spinor as

$$
\phi=\exp \left[i\left(k_{2} x^{2}+k_{3} u-k_{0} \tau\right)\right] \varphi(\xi)
$$

thus we have

$$
\begin{aligned}
& \left(\partial_{\tau}+i k_{3}\right) \varphi_{0}+\left(\frac{k_{2}+k_{0} G}{M}+\partial_{\xi}\right) \varphi_{1}=0 \\
& \left(\partial_{\xi}-\frac{k_{2}+k_{0} G}{M}+\frac{M^{\prime}}{M}\right) \varphi_{0}-2 i k_{0} \varphi_{1}+\left(\partial_{\xi}+\frac{k_{2}+k_{0} G}{M}+\frac{M^{\prime}}{M}\right) \varphi_{3}=0 \\
& \left(\partial_{\xi}-\frac{k_{2}+k_{0} G}{M}\right) \varphi_{1}-\left(k_{0}+k_{3}\right) \varphi_{3}=0
\end{aligned}
$$

the components $\varphi_{0}$ and $\varphi_{3}$ can be expressed in terms of $\varphi_{1}$ by:

$$
\begin{aligned}
& \varphi_{0}=\frac{i}{\left(k_{3}-k_{0}\right)}\left(\partial_{\xi}+\frac{k_{2}+k_{0} G}{M}\right) \varphi_{1} \\
& \varphi_{3}=\frac{i}{\left(k_{3}+k_{0}\right)}\left(\frac{k_{2}+k_{0} G}{M}-\partial_{\xi}\right) \varphi_{1}
\end{aligned}
$$

from the set of above equations it is found that the second order differential equation for $\varphi_{1}$ as

$$
\left\{\partial_{\xi}^{2}+\frac{M^{\prime}}{M} \partial_{\xi}+\frac{k_{3} G^{\prime}}{M}-\left(\frac{k_{2}+k_{0} G}{M}\right)^{2}+k_{0}^{2}-k_{3}^{2}\right\} \varphi_{1}(\xi)=0 .
$$

It is easy to find the other components of the spinor from the differential relations given above. The exact solution will be in the form:

$$
\Psi_{1}=T^{-2}\left(R_{00} \int T^{-1} d x^{0}\right) e^{i\left(k_{2} x^{2}+k_{3} R_{33} x^{3}-i k_{0} R_{00} \int \frac{1}{T} d x^{0}\right)} \varphi_{1}\left(\int R_{11} d x^{1}\right)
$$

\section{The Second Order Form of the Maxwell Equations}

The propagation of electromagnetic fields has been studied for several reasons. There are many astrophysical situations(light deflection in gravitational lensing, pulsars, quasars, black holes) involve strong electromagnetic and gravitational fields in interaction. The interaction of electromagnetic and gravitational fields is described by the Maxwell equations in a given curved background and source. In the absence of electromagnetic source these equations are

$$
\begin{aligned}
& \frac{1}{\sqrt{-g}}\left(\sqrt{-g} F^{\mu \nu}\right)_{, \nu}=0 \\
& F_{\mu \nu, \sigma}+F_{\sigma \mu, \nu}+F_{\nu \sigma, \mu}=0
\end{aligned}
$$

where $F^{\mu \nu}=\partial^{\mu} A-\partial^{\nu} A^{\mu}, A^{\mu}=\left(A^{0}, \vec{A}\right), \vec{A}=\vec{A}(x)$. Here we solve the Maxwell equations for the line element given in (17) to show the correspondence between the mDKP equation and the Maxwell equations. For the particular choice of the functions $T, R_{22}, G$ the solution of the Maxwell equations has been well done by saibatalov[23] 
using similar technique. The contra-variant and covariant field strengths $F^{\mu \nu}$ and $F_{\mu \nu}$ in the general coordinates are

$$
\begin{array}{ll}
F^{01}=T^{-1} E^{(1)}+G(T M)^{-1} B^{(3)} & F_{01}=-T E^{(1)} \\
F^{02}=(T M)^{-1} E^{(2)} & F_{02}=-T M E^{(2)} \\
F^{03}=T^{-1} E^{(3)}-G(T M)^{-1} B^{(1)} & F_{03}=-T E^{(3)} \\
F^{12}=M^{-1} T^{-2} B^{(3)} & F_{12}=G T^{2} E^{(1)}+M T^{2} B^{(3)} \\
F^{13}=-T^{-2} B^{(2)} & F_{13}=-T^{2} B^{(2)} \\
F^{23}=M^{-1} T^{-2} B^{(1)} & F_{23}=-G T^{2} E^{(3)}+M T^{2} B^{(1)}
\end{array}
$$

Here $E^{(i)}$ and $B^{(i)}$ are the components of the electric and magnetic fields in the local Lorentz frame. From equation (51) we find the following coupled equations

$$
\begin{aligned}
& \left(M \partial_{\xi}+M^{\prime}\right) E^{(1)}+\partial_{2} E^{(2)}+M \partial_{u} E^{(3)}-G \partial_{u} B^{(1)}+\left(G \partial_{\xi}+G^{\prime}\right) B^{(3)}=0(54) \\
& \left(M T \partial_{\eta}+2 M \dot{T}\right) E^{(1)}+M \partial_{u} B^{(2)}+\left(G T \partial_{\eta}+2 G \dot{T}-\partial_{2}\right) B^{(3)}=0 \\
& \left(T \partial_{\eta}+2 \dot{T}\right) E^{(2)}-\partial_{u} B^{(1)}+\partial_{\xi} B^{(3)}=0 \\
& -\left(M T \partial_{\eta}+2 M \dot{T}\right) E^{(3)}+\left(G T \partial_{\eta}+2 G \dot{T}-\partial_{2}\right) B^{(1)}+\left(M \partial_{\xi}+M^{\prime}\right) B^{(2)}=0(57)
\end{aligned}
$$

and using equation (52) we get

$$
\begin{aligned}
& \left(G T \partial_{\eta}+2 G \dot{T}-\partial_{2}\right) E^{(1)}+\left(M \partial_{\xi}+M^{\prime}\right) E^{(2)}+\left(M T \partial_{\eta}+2 M \dot{T}\right) B^{(3)}=0(58) \\
& \partial_{u} E^{(1)}-\partial_{\xi} E^{(3)}+\left(T \partial_{\eta}+2 \dot{T}\right) B^{(2)}=0 \\
& G \partial_{u} E^{(1)}-\left(G \partial_{\xi}+G^{\prime}\right) E^{(3)}+\left(M \partial_{\xi}+M^{\prime}\right) B^{(1)}+\partial_{2} B^{(2)}+M \partial_{u} B^{(3)}=0(60) \\
& -M \partial_{u} E^{(2)}-\left(G T \partial_{\eta}+2 G \dot{T}-\partial_{2}\right) E^{(3)}+\left(M T \partial_{\eta}+2 M \dot{T}\right) B^{(1)}=0
\end{aligned}
$$

In terms of the components, these can be written as

$$
\begin{aligned}
& \left(\partial_{\xi}+\frac{M^{\prime}}{M}\right)\left(\begin{array}{c}
E^{(1)} \\
B^{(1)}
\end{array}\right)+\frac{1}{M} \partial_{2}\left(\begin{array}{c}
E^{(2)} \\
B^{(2)}
\end{array}\right)+\frac{G}{M} \partial_{u}\left(\begin{array}{c}
-B^{(1)} \\
E^{(1)}
\end{array}\right) \\
& -\left(\frac{G}{M} \partial_{\xi}+\frac{G^{\prime}}{M}\right)\left(\begin{array}{c}
-B^{(3)} \\
E^{(3)}
\end{array}\right)+\partial_{u}\left(\begin{array}{c}
E^{(3)} \\
B^{(3)}
\end{array}\right)=0 \\
& \left(\partial_{\eta}+\frac{2 \dot{T}}{T}\right)\left(\begin{array}{c}
-B^{(1)} \\
E^{(1)}
\end{array}\right)+\left(\frac{G}{M} \partial_{\eta}+\frac{2 G \dot{T}}{T M}-\frac{1}{T M} \partial_{2}\right)\left(\begin{array}{c}
E^{(3)} \\
B^{(3)}
\end{array}\right) \\
& +\frac{1}{T} \partial_{u}\left(\begin{array}{c}
E^{(2)} \\
B^{(2)}
\end{array}\right)=0 \\
& -\left(\partial_{\eta}+\frac{2 T}{T}\right)\left(\begin{array}{c}
-B^{(2)} \\
E^{(2)}
\end{array}\right)+\frac{1}{T} \partial_{u}\left(\begin{array}{c}
E^{(1)} \\
B^{(1)}
\end{array}\right)-\frac{1}{T} \partial_{\xi}\left(\begin{array}{c}
E^{(3)} \\
B^{(3)}
\end{array}\right)=0 \\
& -\left(\partial_{\eta}+\frac{2 \dot{T}}{T}\right)\left(\begin{array}{c}
-B^{(3)} \\
E^{(3)}
\end{array}\right)+\left(\frac{G}{M} \partial_{\eta}+\frac{2 G \dot{T}}{T M}-\frac{1}{T M} \partial_{2}\right)\left(\begin{array}{c}
E^{(1)} \\
B^{(1)}
\end{array}\right) \\
& +\frac{1}{T}\left(\partial_{\xi}+\frac{M^{\prime}}{M}\right)\left(\begin{array}{c}
E^{(2)} \\
B^{(2)}
\end{array}\right)=0 .
\end{aligned}
$$


If we define new parameter

$$
\tau=\int T^{-1}(\eta) d \eta
$$

and complex spinor as

$$
H=T^{-2}(\tau)\left(\begin{array}{c}
H^{1} \\
H^{2} \\
H^{3}
\end{array}\right)=T^{-2}(\tau)\left(\begin{array}{c}
E^{(1)}+i B^{(1)} \\
E^{(2)}+i B^{(2)} \\
E^{(3)}+i B^{(3)}
\end{array}\right)
$$

the spinor form of the Maxwell equations are found as

$$
\begin{aligned}
& \left(\partial_{\xi}+\frac{M^{\prime}}{M}+\frac{i G}{M} \partial_{u}\right) H^{1}+\frac{1}{M} \partial_{2} H^{2}-i\left(\frac{G}{M} \partial_{\xi}+\frac{G^{\prime}}{M}+i \partial_{u}\right) H^{3}=0 \\
& i \partial_{\tau} H^{1}+\partial_{u} H^{2}+\frac{1}{M}\left(G \partial_{\tau}-\partial_{2}\right) H^{3}=0 \\
& \partial_{u} H^{1}-i \partial_{\tau} H^{2}-\partial_{\xi} H^{3}=0 \\
& \frac{1}{M}\left(G \partial_{\tau}-\partial_{2}\right) H^{1}+\left(\partial_{\xi}+\frac{M^{\prime}}{M}\right) H^{2}-i \partial_{\tau} H^{3}=0 .
\end{aligned}
$$

If the following form of the spinor is used

$$
H^{i}\left(x^{\mu}\right)=\exp \left[i\left(k_{2} x^{2}+k_{3} u-k_{0} \tau\right)\right] N^{i}(\xi) \quad(i=1,2,3)
$$

then we find

$$
\begin{aligned}
& \left(\partial_{\xi}+\frac{M^{\prime}}{M}-\frac{k_{3} G}{M}\right) N^{1}+\frac{i k_{2}}{M} N^{2}-i\left(\frac{G}{M} \partial_{\xi}+\frac{G^{\prime}}{M}-k_{3}\right) N^{3}=0 \\
& k_{0} N^{1}+i k_{3} N^{2}-i \frac{k_{2}+k_{0} G}{M} N^{3}=0 \\
& i k_{3} N^{1}-k_{0} N^{2}-\partial_{\xi} N^{3}=0 \\
& -\frac{k_{2}+k_{0} G}{M} N^{1}+\left(\partial_{\xi}+\frac{M^{\prime}}{M}\right) N^{2}-k_{0} N^{3}=0
\end{aligned}
$$

the components $N^{1}$ and $N^{2}$ can be express in terms of $N^{3}$

$$
\begin{aligned}
& N^{1}=\frac{i}{k_{0}^{2}-k_{3}^{2}}\left[k_{3} \partial_{\xi}+\frac{k_{0}\left(k_{2}+k_{0} G\right)}{M}\right] N^{3} \\
& N^{2}=\frac{1}{k_{3}^{2}-k_{0}^{2}}\left[k_{0} \partial_{\xi}+\frac{k_{3}\left(k_{2}+k_{0} G\right)}{M}\right] N^{3}
\end{aligned}
$$

from the set of above equations it is found that the second order differential equation for $N^{3}$

$$
\left\{\partial_{\xi}^{2}+\frac{M^{\prime}}{M} \partial_{\xi}-\left(\frac{k_{2}+k_{0} G}{M}\right)^{2}+\frac{k_{3} G^{\prime}}{M}-k_{3}^{2}+k_{0}^{2}\right\} N^{3}(\xi)=0 .
$$

This is the same equation (49) and its solution is the same as equation (50). By comparing equations (47)-(48) and (77)-(78) one can obtain the relations between spinor components of the mDKP equation and the Maxwell equations as follow

$$
\varphi_{0}=-N^{1}+i N^{2}, \quad \varphi_{1}=\varphi_{2}=N^{3}, \quad \varphi_{3}=N^{1}+i N^{2}
$$


In terms of the electric and magnetic fields which are given as

$$
E^{(i)}=b_{\mu}^{(0)} b_{\nu}^{(i)} F^{\mu \nu} \quad B^{(i)}=b_{\mu}^{(i)} b_{\nu}^{(0)} \widetilde{F}^{\mu \nu}=\frac{1}{2 \sqrt{-g}} b_{\mu}^{(i)} b_{\nu}^{(0)} \varepsilon^{\mu \nu \alpha \beta} F_{\alpha \beta}
$$

the components of the spinor of the mDKP equation are found that

$$
\begin{aligned}
& \varphi_{0}=i T M\left(F^{02}+i \widetilde{F}^{20}\right)-T\left(F^{01}+i \widetilde{F}^{10}\right)-G T^{2}\left(F^{21}+i \widetilde{F}^{12}\right) \\
& \varphi_{1}=\varphi_{2}=T\left(F^{03}+i \widetilde{F}^{30}\right)+G T^{2}\left(F^{23}+i \widetilde{F}^{32}\right) \\
& \varphi_{3}=i T M\left(F^{02}+i \widetilde{F}^{20}\right)+T\left(F^{01}+i \widetilde{F}^{10}\right)+G T^{2}\left(F^{21}+i \widetilde{F}^{12}\right)
\end{aligned}
$$

\section{The Oscillating Regions of the Photon}

Since it is nor aimed here to solve equation (49) exactly for the given space-times in introduction we will restrict ourselves to discuss how one can obtain the frequency spectrum of the photon by using some examples models of our general space-times. A general method to find the frequency spectrum is to impose the condition on functions which are the solutions of differential equation. The functions obtained must be bounded for all values of as usually done in quantum mechanics, this procedure gives the quantization of frequency. If the function $G$ vanishes the line element (17) reduces an expanding model and one might expect to obtain gravitational red-shift in frequency. But $\mathrm{G}$ is not zero this model represents both expansion and rotation.

We Introduce a new function of the form

$$
\varphi_{1}(\xi)=M^{\frac{1}{2}}(\xi) \chi_{1}(\xi)
$$

using this definition in equation (49) then we get the following form

$$
\left\{\partial_{\xi}^{2}+\frac{M^{\prime \prime}}{2 M}+\left(\frac{M^{\prime}}{2 M}\right)^{2}+\frac{k_{3} G^{\prime}}{M}-\left(\frac{k_{2}+k_{0} G}{M}\right)^{2}+k_{0}^{2}-k_{3}^{2}\right\} \chi_{1}(\xi)=0 .
$$

From this equation we can write,

$$
\omega^{2}(\xi)=\frac{M^{\prime \prime}}{2 M}+\left(\frac{M^{\prime}}{2 M}\right)^{2}+\frac{k_{3} G^{\prime}}{M}-\left(\frac{k_{2}+k_{0} G}{M}\right)^{2}+k_{0}^{2}-k_{3}^{2} .
$$

Case(1):

In the limit of $\xi \rightarrow \infty$ some of the special metrics given in table-1 and table- 2 satisfy $M(\xi)=\infty$. We find $\omega^{2}=k_{0}^{2}-k_{3}^{2}$ and from this result if $\omega^{2}>0$ then we can write $\chi_{1}(\xi)$ as,

$$
\chi_{1}(\xi)=\exp \left[i \sqrt{k_{0}^{2}-k_{3}^{2}} \xi\right]
$$

Case(2):

In the limit of $\xi \rightarrow \infty$ some of the special metrics given in table-1 and table-2 satisfy $M(\xi)=b$. Here $b$ is a constant, then we find $\omega^{2}=k_{0}^{2}-k_{3}^{2}-\left(\frac{k_{2}+a k_{0}}{b}\right)^{2}$. Where $a$ is a constant. From this result if $\omega^{2}>0$ then we can write

$$
\chi_{1}(\xi)=\exp \left[i \sqrt{k_{0}^{2}-k_{3}^{2}-\left(\frac{k_{2}+a k_{0}}{b}\right)^{2}} \xi\right]
$$


Case(3):

In the limit of $\xi \rightarrow 0$ most of the special metrics given in table- 1 and table- 2 satisfy $M(\xi)=c$. Here $c$ is a constant, then we find $\omega^{2}=k_{0}^{2}-k_{3}^{2}-\left(\frac{k_{2}+d k_{0}}{c}\right)^{2}$. Where $d$ is a constant. From this result if $\omega^{2}>0$ then we can write

$$
\chi_{1}(\xi)=\exp \left[i \sqrt{k_{0}^{2}-k_{3}^{2}-\left(\frac{k_{2}+d k_{0}}{c}\right)^{2}} \xi\right]
$$

\section{Results and Discussions}

In the present paper we investigated the mDKP equation and the Maxwell equations in the background of space-time based on the spatially homogenous metric with expansion and rotation which is include many special space-times given in table- 1 and table-2. For each component of the mDKP spinor the corresponding Maxwell field strength tensor components are found. We showed that chargeless and massless spin-1 particle and free space Maxwell equation satisfy the same equations. By using the mDKP equation it was shown that the necessity of $(3+1)$ space-time splitting is not required for electromagnetic fields. This feature strongly motivates us to use the mDKP equation to investigate the behavior of electromagnetic field. Another motivation is that the results obtained can be used to study quantum field theory in curved rotating space-times. Also the wave functions obtained can be used to discuss the Photon production in some special spacetime given in table-1 and table- 2 .

\section{Acknowledgment}

The Authors(MS) would like to thank Turkish Scientific Research Council (Tübitak)Feza Gürsey Institute, Istanbul, for the hospitality his received during him stay in the summer term of 2004.

\section{References}

[1] Tetrode H, Z. Phys. 50, 336 (1928)

[2] Weyl H, Z. Phys. 56, 330 (1929)

[3] Fock V.A, Z. Phys. 57, 261 (1929)

[4] Fock V, Ivanenko D., Compt. Rend. Acad. Sci. 188, 1470 (1929)

[5] Fock V.A, Compt. Rend. Acad. Sci. 189, 25 (1929)

[6] Bade W.L, Jehle H, Rev. Mod. Phys. 25, 714 (1953)

[7] Brill D, Wheeler J, Rev. Mod. Phys. 29, 465 (1957)

[8] Weinberg S, Gravitation and Cosmology: Principles and Applications of the General Theory of Relativity (Wiley, New York, 2002)

[9] Birrel N.D, Davies P.C.W, Quantum Fields in Curved Space (Cambridge University Press, New York, 1982)

[10] Gasperini M, de Sabbata V, Introduction to Gravitation, (World Scientific, 1985)

[11] Good R.H, Phys. Rev. 105, 1914-9 (1957)

[12] Moses H.E, Nuovo Cim. Suppl. 7, 1-18 (1958), Phys. Rev. 113, 1670-9 (1959) 
[13] Petiau G, Acad.R, Belg.Cl. Sci.Mem.Collet. (8) 16, No.2(1936); Duffin R.J, Phys.Rev. 54, 1114 (1938); Kemmer N, Proc.Roy.Soc. (London) A173, 91 (1939);Kemmer N, Proc. Camb. Phil. Soc. 39, 189-96 (1943); Taketani M and Sakata S, Proc. Phys. Math. Soc. Japan 22, 757-70 (1940) [Reprinted in Suppl.Progr.Theor.Phys. 22, 84 (1955)]

[14] Krajcik R.A and Nieto M.M, Am. J. Phys. 45, 818 (1977)

[15] Gribov V, Eur. Phys. J. C10, 71 (1999)

[16] Kanatchikov I.V, Rept. Math. Phys. 46, 107 (2000)

[17] Red'kov V.M, arXiv : quant-ph/9812007(1998)

[18] Lunardi J.T, Pimentel B.M and Teixera R.G, in Geometrical Aspects of Quantum Fields, proceedings of the 2000 Londrina Workshop, Londrina, Brazil, edited by Bytsenko A.A, Golcalves A.E and Pimentel B.M; World Scientific, Singapore, (2001)

[19] Ünal N, Found. Phys. 27, 795 (1997)

[20] Ünal N, Sucu Y, Int. J. Mod. Phys. A17, 1137-1147 (2002)

[21] Havare A, Yetkin T, Class. Quantum Grav. 19, 2783-2791 (2002)

[22] Havare A, Yetkin T, Int. J. of Mod. Phys. A (Accepted)

[23] Saibabalov R.X, Gen. Rel. and Grav. 27, 697 (1995)

[24] Gamow G., Nature (London) 158, 549 (1946)

[25] Gödel K., Rev. Mod. Phys. 21, 447 (1949)

[26] Havare A, Yetkin T, Chinese J. of Phys., Vol.41, No.5 (2003)

[27] Gron O, Soleng H.H, Acta Physica Polonica No:7 Vol.B20 (1989)

[28] Carneiro S, Phys. Rev. D 61 (2000)

[29] Garcia de Andrade L.C, Gen.Rel.Grav. 35, 1279-1283 (2003)

[30] Klein C, Theor.Math.Phys. 127 767-778; Teor.Mat.Fiz., 127,418-431(2001)

[31] Fagundes H.V, Gen. Rel. and Cos., Vol.24, No:2 (1992)

[32] Gibbons G.W, Herdeiro C.A.R, Class.Quant.Grav. 18, 1677-1690 (2001)

[33] Herrera L, Santos N.O, Class.Quant.Grav. 18, 3847-3856 (2001)

[34] Avakyan R.M, Chubaryan E.V Yeranyan A.H, arXiv:gr-qc-0102030 (2001)

[35] Dao-Jun Lii , Xin Zhou Li, Chin.Phys.Lett. 20, 1678 (2003)

[36] Abramowitz M and Stegun I, Handbook of Mathematical Functions, (New York, 1974)

[37] Cohen J.M, Vishveshwara and Dhurandhar S. V., J. Phys. 13, 933 (1980)

[38] Pimentel Luis O and Macias A, Physics Letters A Vol 7, 11, 325 (1986)

[39] MacCallum M.A.H, arXiv:gr-qc-9212013 (1992) 